International Journal of Pure and Applied Mathematics

Volume 114 No. 4 2017, 895-906

ISSN: 1311-8080 (printed version); ISSN: 1314-3395 (on-line version)

url: http://www.ijpam.eu

doi: 10.12732 /ijpam.v114i4.19

\title{
ON COVERING PROPERTIES IN TERMS OF GENERALIZED FORM OF PREOPEN SETS
}

\author{
M.K. Ghosh \\ Department of Mathematics \\ Kalyani Mahavidyalaya \\ Kalyani, 741235, Nadia, West Bengal, INDIA
}

\begin{abstract}
In this paper, we introduce the concept of a new class of sets viz. $\hat{\gamma}$-preopen sets using an operation $\gamma$ to preopen sets. This class of sets contains the class of preopen sets. Such sets are used to introduce and study some separation as well as covering axioms.
\end{abstract}

AMS Subject Classification: 54A05, 54A10, 54C10, 54D20

Key Words: preopen, $\hat{\gamma}$-preopen, $\hat{\gamma}$-strongly compact, $\hat{\gamma}$-p-closed

\section{Introduction and Preliminaries}

Many classical topological notions such as continuity of functions, compactness and connectedness have been extended by using preopen sets replacing the role of open sets. From the literature, we see that the study of topological properties via preopenness has gained significant importance in General Topology. In this paper, we used operation approach on preopen sets in a different way to introduce a new kind sets known as $\hat{\gamma}$-preopen sets, which contains the class of preopen [16] sets in a topological space. The concept of operation in a topological was first introduced by Kasahara [12] and it was further investigated by Jankovic [10]. Then, Ogata [19] defined the concept of $\gamma$-operation in a topological space and consequently introduced $\gamma$-open sets.

By a space $X$ we denote a topological space $(X, \tau)$ without any separation axiom. For a subset $A$ of $X$, we denote by $\operatorname{int}(A)$ and $\operatorname{cl}(A)$ the interior and clo-

$\begin{array}{lr}\text { Received: } & \text { April 15, } 2017 \\ \text { Revised: } & \text { May 20, 2017 } \\ \text { Published: } & \text { June 7, 2017 }\end{array}$

(c) 2017 Academic Publications, Ltd. url: www.acadpubl.eu 
sure of $A$ respectively. A subset $A$ of $X$ is called preopen [16] if $A \subset \operatorname{int}(\operatorname{cl}(A))$. Set of all preopen sets in $X$ is denoted by $P O(X)$ and that of containing a point $x$ of $X$, by $P O(X, x)$. Let $A$ be a subset of space $X$, then pre-interior of $A$, denoted by $\operatorname{pint}(A)$ and defined as the union of all preopen sets contained in $A$ and pre-closure of $A$ denoted by $\operatorname{pcl}(A)$ and defined as the intersection of all preclosed sets containing $A$. A topological space $(X, \tau)$ is called submaximal [2] if every dense subset of $X$ is open equivalently, every preopen set is open. A topological space $X$ is said to be strongly compact [3] if every cover of $X$ by preopen sets admits a finite subcover. A topological space $X$ is said to be p-closed [1] if for every preopen cover $\left\{V_{\alpha}: \alpha \in \Lambda\right\}$ of $X$, there exist a finite subset $\Lambda_{0}$ of $\Lambda$ such that $X=\left\{p c l\left(V_{\alpha}\right): \alpha \in \Lambda_{0}\right\}$. Let $A$ be a subset of a topological space $X$, then $\theta$-closure of $A$ (resp. pre- $\theta$-closure of $A$ ) denoted by $\theta-c l(A)$ (resp. $\operatorname{pcl}_{\theta}(A)$ ) is defined as $\cup\{x \in X: \operatorname{cl}(U) \cap A \neq \emptyset\}$ (resp. $\cup\{x \in X: \operatorname{pcl}(U) \cap A \neq \emptyset\})$. A filter base $\mathcal{F}$ on a topological space $(X, \tau)$ is said to pre- $\theta$-converge [8] to a point $x \in X$ if for each $V \in P O(X, x)$, there exists $F \in \mathcal{F}$ such that $F \subset \operatorname{pcl}(V)$. A filter base $\mathcal{F}$ is said to pre- $\theta$-accumulate [8] at $x \in X$ if $\operatorname{pcl}(V) \cap F \neq \emptyset$ for every $V \in P O(X, x)$ and every $F \in \mathcal{F}$. A topological space $(X, \tau)$ is said to be strongly p-regular [8] (resp. p-regular [6]) if for each point $x \in X$ and each preclosed set (resp. closed set) $F$ such that $x \notin F$, there exist disjoint preopen sets $U$ and $V$ such that $x \in U$ and $F \subset V$. A topological space $(X, \tau)$ is called strongly normal [17] if for each pair of disjoint preclosed sets $\mathrm{A}$ and $\mathrm{B}$ of $\mathrm{X}$, there exist disjoint preopen sets $\mathrm{U}$ and $\mathrm{V}$ containing them. A function $f:(X, \tau) \rightarrow\left(Y, \tau^{\prime}\right)$ is called preirresolute [20] if $f^{-1}(V)$ is preopen in $X$ for every preopen subset $V$ of $Y$.

An operation $\gamma[19]$ on a topology $\tau$ on $X$ is a mapping $\gamma: \tau \rightarrow P(X)$, such that $V \subset V^{\gamma}$ for each $V \in \tau$, where $P(X)$ is the power set of $X$ and $V^{\gamma}$ denotes the value of $\gamma$ at $V$. A subset $A$ of $X$ with an operation $\gamma$ on $\tau$ is called $\gamma$-open [19] if for each $x \in A$, there exists an open set $U$ such that $x \in U$ and $U^{\gamma} \subset A$. $\tau_{\gamma}$ denotes the set of all $\gamma$-open sets in $X$. Clearly $\tau_{\gamma} \subset \tau$. The $\tau_{\gamma}$-closure [19] of subset $A$ of $X$ is denoted by $\tau_{\gamma}-\operatorname{cl}(A)$ and is defined to be the intersection of all $\gamma$-closed sets containing $S$ and $\tau_{\gamma}$-interior [13] of $A$ is denoted by $\tau_{\gamma}$-int $(A)$ and is defined as the union of all $\gamma$-open sets of $X$ contained in $A$. A topological $X$ with an operation $\gamma$ on $\tau$ is said to be $\gamma$-regular [19] if for each $x \in X$ and open neighborhood $V$ of $x$, there exists an open neighborhood $U$ of $x$ such that $U^{\gamma}$ is contained in $V$. Let $(X, \tau)$ and $\left(Y, \tau^{\prime}\right)$ be two topological spaces and $\gamma$ be an operation on $\tau$. Then a function $f:(X, \tau) \rightarrow\left(Y, \tau^{\prime}\right)$ is said to be $\gamma$-continuous [5] at $x$ if for each open set $V$ containing $f(x)$, there exists an $\gamma$-open set $U$ in $X$ containing the point $x$ such that $f(U) \subset V$.

In rest of this article by spaces $X$ and $Y$ we will denote topological spaces 
$(X, \tau)$ and $\left(Y, \tau^{\prime}\right)$ with operations $\gamma$ on $\tau$ and $\gamma^{\prime}$ on $\tau^{\prime}$ respectively. By a function $f: X \rightarrow Y$ we mean a function $f:(X, \tau) \rightarrow\left(Y, \tau^{\prime}\right)$ with operations $\gamma$ on $\tau$ and $\gamma^{\prime}$ on $Y$ respectively.

\section{2. $\hat{\gamma}$-Preopen Sets}

Definition 2.1. A subset $A$ of a topological space $X$, is called $\hat{\gamma}$-preopen set if $A \subset \operatorname{int}\left(\tau_{\gamma}-\operatorname{cl}(A)\right)$. The complement of a $\hat{\gamma}$-preopen set is called $\hat{\gamma}_{-}$ preclosed set.

The family of all $\hat{\gamma}$-preopen sets (resp. $\hat{\gamma}$-preclosed) sets of $X$ is denoted by $\hat{\gamma}-P O(X)$ (resp. $\hat{\gamma}-P C(X))$. Clearly $\tau_{\gamma} \subset \hat{\gamma}-P O(X) \subset \tau$ and $P O(X) \subset \hat{\gamma}^{-}$ $P O(X) \subset \tau$.

The following example shows that the above inclusions are proper in general.

Example 2.2. Let $X=\{a, b, c\}$ and $\tau=\{\emptyset,\{a\},\{b\},\{a, b\},\{a, c\}, X\}$ is a topology on $X$. Define an operation $\gamma$ on $\tau$ by

$$
A^{\gamma}= \begin{cases}A & \text { if } b \in A \\ \operatorname{cl}(\mathrm{A}) & \text { if } b \notin A\end{cases}
$$

Then $\{c\}$ and $\{b, c\}$ are neither preopen nor $\gamma$-open but $\hat{\gamma}$-preopen sets.

Remark 2.3. If a topological space is $\gamma$-regular [19], then $\tau_{\gamma}=\tau$ and therefore $P O(X)=\hat{\gamma}-P O(X)$.

Definition 2.4. A space $X$ is said to satisfy a property $\gamma$-p [4] if $\tau_{\gamma^{-}}$ $\operatorname{cl}(A \cap B)=\tau_{\gamma^{-}} c l(A) \cap \tau_{\gamma^{-}} c l(B)$.

Theorem 2.5. (a) Union of arbitrary family of $\hat{\gamma}$-preopen sets is $\hat{\gamma}$ preopen.

(b) If a topological space satisfies the property $\gamma-p$, then the intersection of any two $\hat{\gamma}$-preopen sets is a $\hat{\gamma}$-preopen set and $\hat{\gamma}-P O(X)$ forms a topology finer that $\tau$.

Definition 2.6. A topological space $X$ is said to be $\hat{\gamma}$-submaximal every $\hat{\gamma}$-preopen set in $\gamma$-open.

Theorem 2.7. If a topological space $X$ is $\hat{\gamma}$-submaximal then $\hat{\gamma}-P O(X)$ forms a topology coarser than $\tau$.

Remark 2.8. The converse of the above theorem is not true. 
Example 2.9. Let $X=\{a, b, c\}$ and $\tau=\{\emptyset,\{a\},\{b\},\{a, b\},\{a, c\}, X\}$ is a topology on $X$. Define an operation $\gamma$ on $\tau$ by

$$
A^{\gamma}= \begin{cases}A & \text { if } b \in A \\ \operatorname{cl}(\mathrm{A}) & \text { if } b \notin A\end{cases}
$$

Then $\hat{\gamma}-P O(X)$ forms a topology but $X$ is not $\hat{\gamma}$-submaximal.

Definition 2.10. Let $A$ be a subset of a topological space $X$. Then $\hat{\gamma}$-preinterior (resp. $\hat{\gamma}$-pre-closure) of $A$, denoted by $\hat{\gamma}$-pint $(A)(\operatorname{resp} . \hat{\gamma}-\operatorname{pcl}(A))$ and is defined as the union of all $\hat{\gamma}$-preopen sets contained in $A$ (resp. intersection of all $\hat{\gamma}$-preclosed sets containing $A$.

We now state the following theorem without proof, which will be used in the consequent sections.

Theorem 2.11. For any subsets $A$ and $B$ of a topological space $X$, the following holds:

(a) $x \in \hat{\gamma}-p c l(A)$ if and only if $A \cap U \neq \emptyset$ for every $U \in \hat{\gamma}-P O(X, x)$.

(b) $A$ is $\hat{\gamma}$-preopen (resp. $\hat{\gamma}$-preclosed) if and only if $A=\hat{\gamma}$-pint $(A)$ (resp. $A=\hat{\gamma}-\operatorname{pcl}(A))$.

(c) If $A \subset B$ then $\hat{\gamma}-\operatorname{pint}(A) \subset \hat{\gamma}-\operatorname{pint}(B)$ and $\hat{\gamma}-\operatorname{pcl}(A) \subset \hat{\gamma}-\operatorname{pcl}(B)$.

(d) $\hat{\gamma}-\operatorname{pint}(A)=X-\hat{\gamma}-p c l(B)$.

(e) $\tau_{\gamma}-\operatorname{int}(A) \subset \operatorname{int}(A) \subset \operatorname{pint}(A) \subset \hat{\gamma}-\operatorname{pint}(A)$ and $\gamma-\operatorname{pint}(A) \subset \hat{\gamma}-\operatorname{pint}(A)$.

(f) $\hat{\gamma}-\operatorname{pcl}(A) \subset \operatorname{pcl}(A) \subset \operatorname{cl}(A) \subset \tau_{\gamma}-c l(A)$ and $\hat{\gamma}-p c l(A) \subset \gamma-p c l(A)$.

Definition 2.12. A point $x \in X$ is said to be a $\hat{\gamma}$-pre- $\theta$-accumulation point of a subset $A$ of a topological space $X$ if $\hat{\gamma}-p c l(U) \cap A \neq \emptyset$ for every $U \in \hat{\gamma}-P O(X, x)$.

The set of all $\hat{\gamma}$-pre- $\theta$-accumulation points of a subset $A$ of $X$ is called $\hat{\gamma}$-pre- $\theta$-closure of $A$ and is denoted by $\hat{\gamma}-p c l_{\theta}(A)$.

A subset $A$ of a topological space $X$ is said to be $\hat{\gamma}$-pre- $\theta$-closed if $\hat{\gamma}$ $\operatorname{pcl}_{\theta}(A)=A$. The complement of a $\hat{\gamma}$-pre- $\theta$-closed set is called $\hat{\gamma}$-pre- $\theta$-open set.

Lemma 2.13. A subset $A$ of a space $X$ is $\hat{\gamma}$-pre- $\theta$-open if and only if for each $x \in A$, there exists a $V \in \hat{\gamma}-P O(X, x)$ such that $\hat{\gamma}-p c l(V) \subset A$.

Proof. Let $A$ be a $\hat{\gamma}$-pre- $\theta$-open and $x \in A$. Then $(X-A)$ is $\hat{\gamma}$-pre- $\theta$ closed set and so for each $x \in A$, there exists $V \in \hat{\gamma}-P O(X, x)$ such that $\hat{\gamma}-p c l(V) \cap(X-A)=\emptyset$ and therefore $\hat{\gamma}-p c l(V) \subset A$.

Conversely, if the condition does not hold, there exists $x \in A$ such that $\hat{\gamma}-p c l(V) \not \subset A$ for all $V \in \hat{\gamma}-P O(X, x)$. This implies $\hat{\gamma}-p c l(V) \cap(X-A) \neq \emptyset$ 
for all $V \in \hat{\gamma}-P O(X, x)$ and so $x$ is a $\hat{\gamma}$-pre- $\theta$-accumulation point of $(X-A)$. Hence $(X-A)$ is not $\hat{\gamma}$-pre- $\theta$-closed.

Theorem 2.14. Let $A$ and $B$ by any two subsets a space $X$. Then the following properties hold:

(a) Every $\theta$-closed and pre- $\theta$-closed sets are $\hat{\gamma}$-pre- $\theta$-closed.

(b) $\hat{\gamma}-p c l_{\theta}(A) \subset \operatorname{pcl}_{\theta}(A) \subset \theta-c l(A)$.

(c) If $A \subset B$ then $\hat{\gamma}-p c l_{\theta}(A) \subset \hat{\gamma}-p c l_{\theta}(B)$.

(d) Intersection of arbitrary family of $\hat{\gamma}$-preclosed sets is $\hat{\gamma}$-preclosed set in $X$.

Proof. Straightforward.

\section{Separation Axioms}

In this section we focus our attention to the introduction of certain separation axioms utilizing the concepts developed in the earlier section and discuss some of their characterizations.

Definition 3.1. A space $X$ is called

(a) $\hat{\gamma}$-pre- $T_{0}$ if and only if for each pair of distinct points $x, y \in X$, there exists an $U \in \hat{\gamma}-P O(X, x)$ such that either $x \in U$ and $y \notin U$ or $x \notin X$ and $y \in U$.

(b) $\hat{\gamma}$-pre- $T_{1}$ if and only if each pair of distinct points $x, y \in X$, there exists two $\hat{\gamma}$-preopen sets $U, V$ such that $x \in U$ but $y \notin U$ and $y \in V$ but $x \notin V$.

(c) $\hat{\gamma}$-pre- $T_{2}$ if for each pair of distinct points $x, y \in X$, there exists $U \in \hat{\gamma}$ $P O(X, x)$ and $V \in \hat{\gamma}-P O(X, y)$ such that $U \cap V=\emptyset$.

(d) $\hat{\gamma}$-pre-Urysohn if for each pair of distinct points $x, y \in X$, there exists $U \in \hat{\gamma}-P O(X, x)$ and $V \in \hat{\gamma}-P O(X, y)$ such that $\hat{\gamma}-p c l(U) \cap \hat{\gamma}-p c l(V)=\emptyset$.

Definition 3.2. A space $X$ is called

(e) $\hat{\gamma}$-strongly pre-regular if for each point $x \in X$ and each $\hat{\gamma}$-preclosed set $F$ not containing $x$, there exist disjoint $\hat{\gamma}$-preopen sets $U$ and $V$ such that $x \in U$ and $F \subset V$.

(f) $\hat{\gamma}$-strongly pre-normal if for any pair of disjoint $\hat{\gamma}$-preclosed sets $A, B$ of $X$, there exists disjoint $\hat{\gamma}$-preopen sets $U$ and $V$ such that $A \subset U$ and $B \subset V$.

Remark 3.3. (a) The family of pre- $T_{0}$ [11] (resp. pre- $T_{1}$ [11], pre- $T_{2}$ [11], strongly pre-regular [6], strongly pre-normal [17]) spaces are contained in the family of $\hat{\gamma}-T_{0}$ (resp. $\hat{\gamma}$-pre- $T_{1}, \hat{\gamma}$-pre- $T_{2}, \hat{\gamma}$-strongly pre-regular, $\hat{\gamma}$-strongly pre-normal) spaces.

(b) The family of Urysohn [22] and pre-Urysohn spaces [15] are contained in 
$\hat{\gamma}$-pre-Urysohn spaces.

(c) The family of $\hat{\gamma}$-pre-Urysohn spaces is contained in the family of $\hat{\gamma}$-pre- $T_{2}$ spaces and the family of $\hat{\gamma}$-pre- $T_{2}$ spaces is contained in the family of $\hat{\gamma}$-pre- $T_{1}$ spaces.

The following three theorems characterizes $\hat{\gamma}$-pre- $T_{1}, \hat{\gamma}$-pre- $T_{2}$ and $\hat{\gamma}$-strongly pre-regular spaces respectively. Their proofs being easy to check are omitted.

Theorem 3.4. A space topological $X$ is $\hat{\gamma}-$ pre- $T_{1}$ if and only if singleton sets are $\hat{\gamma}$-preclosed.

Theorem 3.5. For a topological spaces $X$, the following statements are equivalent:

(a) $X$ is $\hat{\gamma}$-pre- $T_{2}$;

(b) For each distinct points $x, y$ of $X$, there exist $U \in \hat{\gamma}-P O(X, x)$ such that $y \notin \hat{\gamma}-p c l(U)$;

(c) For each $x \in X, \cap\{\hat{\gamma}-p c l(U): U \in \hat{\gamma}-P O(X, x)\}=\{x\}$.

Theorem 3.6. A space $X$ is $\hat{\gamma}$-strongly pre-regular if and only if for each $x \in X$ and each $U \in \hat{\gamma}-P O(X, x)$, there exists a $V \in \hat{\gamma}-P O(X, x)$ such that $\hat{\gamma}-\operatorname{pcl}(V) \subset U$.

Theorem 3.7. Every $\hat{\gamma}$-strongly pre-regular and $\hat{\gamma}$-pre- $T_{2}$ spaces is $\hat{\gamma}$-preUrysohn.

Proof. Let $x$ and $y$ be two distinct points in a $\hat{\gamma}$-strongly pre-regular, $\hat{\gamma}$ pre- $T_{2}$ space $X$. Now, since $X$ is $\hat{\gamma}$-pre- $T_{2}$, there exists $U \in \hat{\gamma}-P O(X, x)$ and $V \in \hat{\gamma}-P O(X, y)$ such that $U \cap V=\emptyset$ and $\hat{\gamma}-p c l(U) \cap V=\emptyset$. Then $U^{\prime}=$ $X-\hat{\gamma}-\operatorname{pcl}(U) \in \hat{\gamma}-P O(X, y)$ and so by above Theorem 3.6, there exists $V^{\prime} \in \hat{\gamma}$ $P O(X, y)$ such that $\hat{\gamma}-p c l\left(V^{\prime}\right) \subset U^{\prime}$ and therefore $\hat{\gamma}-\operatorname{pcl}\left(V^{\prime}\right) \cap \hat{\gamma}-p c l(U)=\emptyset$. Hence $X$ is $\hat{\gamma}$-pre-Urysohn.

Definition 3.8. A function $f: X \rightarrow Y$ is said to be $\left(\hat{\gamma}, \hat{\gamma}^{\prime}\right)$-preopen if image of each $\hat{\gamma}$-preopen set is $\hat{\gamma}^{\prime}$-preopen.

Definition 3.9. A function $f: X \rightarrow Y$ is said to be $\left(\hat{\gamma}, \hat{\gamma}^{\prime}\right)$-pre-irresolute (resp. quasi $\left(\hat{\gamma}, \hat{\gamma}^{\prime}\right)$-pre-irresolute) if for each $x \in X$ and for each $\hat{\gamma}^{\prime}$-preopen set $V$ containing $f(x)$, there exists an $\hat{\gamma}$-preopen set $U$ containing $x$ such that $f(U) \subset V\left(\operatorname{resp} . f(U) \subset \hat{\gamma}^{\prime}-p c l(V)\right)$.

Theorem 3.10. Let $f: X \rightarrow Y$ be a quasi $\left(\hat{\gamma}, \hat{\gamma}^{\prime}\right)$-pre-irresolute injection and $Y$ be a $\hat{\gamma}^{\prime}$-pre-Urysohn. Then $X$ is $\hat{\gamma}$-pre- $T_{2}$.

Proof. Let $x$ and $y$ be any two distinct points of $X$. Since $f$ is an injection and $Y$ is $\hat{\gamma}^{\prime}$-pre-Urysohn, there exist $V_{x} \in \hat{\gamma}^{\prime}-P O(Y, f(x))$ and $V_{y} \in \hat{\gamma}^{\prime}$ - 
$P O(Y, f(y))$ such that $\hat{\gamma}^{\prime}-p c l\left(V_{x}\right) \cap \hat{\gamma}^{\prime}-p c l\left(V_{y}\right)=\emptyset$. Also, $f$ being quasi $\left(\hat{\gamma}, \hat{\gamma}^{\prime}\right)$ pre-irresolute function, there exists $U_{x} \in \gamma-P O(X, x)$ and $U_{y} \in \gamma-P O(X, y)$ such that $f\left(U_{x}\right) \subset \hat{\gamma}^{\prime}-p c l\left(V_{x}\right)$ and $f\left(U_{y}\right) \subset{\hat{\gamma^{\prime}}}^{\prime} p c l\left(V_{y}\right)$. Then $f\left(U_{x}\right) \cap f\left(U_{y}\right)=\emptyset$ and so $U_{x} \cap U_{y}=\emptyset$. Therefore, $X$ is $\hat{\gamma}-$ pre- $T_{2}$.

Theorem 3.11. Let $f: X \rightarrow Y$ be an $\left(\hat{\gamma}, \hat{\gamma}^{\prime}\right)$-preopen bijection and $X$ be $\hat{\gamma}$-pre-Urysohn. Then $Y$ is $\hat{\gamma}^{\prime}$-pre-Urysohn.

Proof. Let $y_{1}$ and $y_{2}$ be any two distinct points of $Y$. Since $f$ is a bijection, there exists distinct points $x_{1}, x_{2} \in X$ such that $f\left(x_{1}\right)=y_{1}$ and $f\left(x_{2}\right)=y_{2}$. Again, since $f$ is $\hat{\gamma}$-pre-Urysohn, there exists $U_{x_{1}} \in \hat{\gamma}-P O\left(X, x_{1}\right)$ and $U_{x_{2}} \in \hat{\gamma}$ $P O\left(X, x_{2}\right)$ such that $\hat{\gamma}-p c l\left(U_{x_{1}}\right) \cap \hat{\gamma}-p c l\left(U_{x_{2}}\right)=\emptyset$. Now, $f$ being $\left(\hat{\gamma}, \hat{\gamma}^{\prime}\right)$-preopen bijection, $f\left(X-\hat{\gamma}-p c l\left(U_{x_{1}}\right)\right)=Y-f\left(\hat{\gamma}-p c l\left(U_{x_{1}}\right)\right)$ and $f\left(X-\hat{\gamma}-p c l\left(U_{x_{2}}\right)\right)=$ $Y-f\left(\hat{\gamma}-p c l\left(U_{x_{2}}\right)\right)$ are $\hat{\gamma}^{\prime}$-preopen sets and so $f\left(\hat{\gamma}-p c l\left(U_{x_{1}}\right)\right)$ and $f\left(\hat{\gamma}-p c l\left(U_{x_{2}}\right)\right)$ are $\hat{\gamma}^{\prime}$-preclosed sets. Therefore, $\hat{\gamma}^{\prime}-p c l\left(f\left(U_{x_{1}}\right)\right) \cap \hat{\gamma}^{\prime}-p c l\left(f\left(U_{x_{2}}\right)\right) \subset \hat{\gamma}^{\prime}-p c l(f(\hat{\gamma}-$ $\operatorname{pcl}\left(\left(U_{x_{1}}\right)\right) \cap \hat{\gamma}^{\prime}-\operatorname{pcl}\left(f\left(\hat{\gamma}-p c l\left(\left(U_{x_{2}}\right)\right)=f\left(\hat{\gamma}^{\prime}-p c l\left(U_{x_{1}}\right)\right) \cap f\left(\hat{\gamma}^{\prime}-p c l\left(U_{x_{2}}\right)\right)=f(\hat{\gamma}-\right.\right.$ $\left.\operatorname{pcl}\left(U_{x_{1}}\right) \cap \hat{\gamma}-\operatorname{pcl}\left(U_{x_{2}}\right)\right)=\emptyset$. Since $f$ is $\left(\hat{\gamma}, \hat{\gamma}^{\prime}\right)$-preopen, $f\left(U_{x_{1}}\right) \in \hat{\gamma}^{\prime}-P O\left(Y, y_{1}\right)$ and $f\left(U_{x_{2}}\right) \in \hat{\gamma}^{\prime}-P O\left(Y, y_{2}\right)$ with $\hat{\gamma}^{\prime}-p c l\left(f\left(U_{x_{1}}\right)\right) \cap \hat{\gamma}^{\prime}-p c l\left(f\left(U_{x_{2}}\right)\right)=\emptyset$. Hence $Y$ is $\hat{\gamma}^{\prime}$-pre-Urysohn.

\section{Covering Axioms}

Definition 4.1. A subset $S$ of a space $X$ is called $\hat{\gamma}$-strongly compact (resp. $\hat{\gamma}$-p-closed) relative to $X$ if every cover of $S$ by $\hat{\gamma}$-preopen sets of $X$ has a finite subfamily whose members (resp. $\hat{\gamma}$-preclosures) cover $S$.

A space $X$ is called $\hat{\gamma}$-strongly compact (resp. $\hat{\gamma}$-p-closed) if $X$ is $X$ is $\hat{\gamma}$-strongly compact (resp. $\hat{\gamma}$-p-closed) relative to $X$.

Remark 4.2. It is clear that $\hat{\gamma}$-strongly compactness implies $\hat{\gamma}$-p-closedness and $\hat{\gamma}$-strongly compactness (resp. $\hat{\gamma}$-p-closedness) implies strongly compactness [18] (resp. p-closedness [1]). Consequently we have, $\hat{\gamma}$-strongly compact $\Rightarrow$ strongly compact $\Rightarrow \alpha$-compact $[14] \Rightarrow$ compact $\Rightarrow$ nearly compact $[21] \Rightarrow$ QHC.

Theorem 4.3. If $X$ is space such that every $\hat{\gamma}$-preopen subset of $X$ is $\hat{\gamma}$-strongly compact relative to $X$, then every subset of $X$ is $\hat{\gamma}$-strongly compact relative to $X$.

Proof. Let $S$ be an arbitrary subset of $X$ and $\left\{V_{i}: i \in I\right\}$ be a cover of $S$ by $\hat{\gamma}$-preopen sets of $X$. Then the family $\left\{V_{i}: i \in I\right\}$ ia $\hat{\gamma}$-preopen cover of 
the $\hat{\gamma}$-preopen set $\cup\left\{V_{i}: i \in I\right\}$. Hence by hypothesis there is finite subfamily $\left\{V_{i_{\mathrm{j}}}: j \in \mathbb{N}_{0}\right\}$ which covers $\cup\left\{V_{i}: i \in I\right\}$, where $\mathbb{N}_{0}$ is finite subset of the naturals $\mathbb{N}$. This subfamily is also a cover of the set $S$. Hence the theorem.

Definition 4.4. A filter base $\mathcal{F}$ on a topological space $X$ is said to be $\hat{\gamma}$-pre-converge (resp. $\hat{\gamma}$-pre- $\theta$-converge) to a point $x \in X$ if for each $V \in \hat{\gamma}$ $P O(X, x)$, there exists an $F \in \mathrm{F}$ such that $F \subset V(\operatorname{resp} . F \subset \hat{\gamma}-p c l(V))$.

A filter base $\mathcal{F}$ on a topological space $X$ is said to be $\hat{\gamma}$-pre-accumulate (resp. $\hat{\gamma}$-pre- $\theta$-accumulate) at $x \in X$ if $V \cap F \neq \emptyset$ (resp. $\hat{\gamma}$-pcl $(V) \cap F \neq \emptyset$ ) for every $V \in \hat{\gamma}-P O(X, x)$ and every $F \in \mathrm{F}$.

Theorem 4.5. The following conditions are equivalent for a topological space $X$ :

(a) $X$ is $\hat{\gamma}$-p-closed;

(b) every ultrafilter base $\hat{\gamma}$-pre- $\theta$-converges at some point of $X$;

(c) every filter base $\hat{\gamma}$-pre- $\theta$-accumulates at some point of $X$;

(d) for every family $\left\{V_{\alpha}: \alpha \in \Lambda\right\}$ of $\hat{\gamma}$-preclosed subsets such that $\cap\left\{V_{\alpha}: \alpha \in\right.$ $\Lambda\}=\emptyset$, there exist $\alpha_{1}, \alpha_{2}, \ldots, \alpha_{n} \in \Lambda$ such that $\cap_{i=1}^{n} \hat{\gamma}-\operatorname{pint}\left(V_{\alpha_{\mathrm{i}}}\right)=\emptyset$.

Proof. (a) $\Rightarrow(\mathrm{b})$ : Suppose $X$ is $\hat{\gamma}$-p-closed and $\Omega$ is an ultrafilter base on $X$ which does not $\hat{\gamma}$-pre- $\theta$-converges to any point of $X$. Now, $\Omega$ being an ultrafilter base on $X$, it can not $\hat{\gamma}$-pre- $\theta$-accumulate at any point of $X$. Then for each $x \in X$, there is an $F_{x} \in \Omega$ and a $V_{x} \in \hat{\gamma}-P O(X, x)$ such that $\hat{\gamma}-p c l\left(V_{x}\right) \cap F_{x}=\emptyset$ and so the family $\left\{V_{x}: x \in X\right\}$ forms a cover of $X$ by $\hat{\gamma}$-preopen sets. Now, $X$ being $\hat{\gamma}$-p-closed, there exist finite number of points $x_{1}, x_{2}, \ldots, x_{n} \in X$ such that $X=\cup_{i=1}^{n} \hat{\gamma}-p c l\left(V_{x_{\mathrm{i}}}\right)$. Again since $\Omega$ is a filter base on $X$, there exists an $F_{0} \in \Omega$ such that $F_{0} \subset \cap_{i=1}^{n} F_{x_{\mathrm{i}}}$ and thus $F_{0}=\emptyset$ - a contradiction.

(b) $\Rightarrow(\mathrm{c})$ : Let $\mathcal{F}$ be any filter base on $X$. Then there is an ultrafilter base $\Omega$ containing $\mathcal{F} \hat{\gamma}$-pre- $\theta$-converging to some point of $x \in X$. We take any $V \in \hat{\gamma}$ $P O(X, x)$ and $F \in \mathcal{F}$, then there exists an $F^{\prime} \in \Omega$ such that $F^{\prime} \subset \hat{\gamma}-p c l(V)$ and $F \cap F^{\prime} \neq \emptyset$. Therefore, $\emptyset \neq F \cap F^{\prime} \subset \hat{\gamma}-p c l(V) \cap F$ and so the filter base $\mathcal{F}$, $\hat{\gamma}$-pre- $\theta$-accumulates at $x \in X$.

$(\mathrm{c}) \Rightarrow(\mathrm{d})$ : Let $\left\{V_{\alpha}: \alpha \in \Delta\right\}$ be a family of $\hat{\gamma}$-preclosed subsets of $X$ with $\left\{V_{\alpha}: \alpha \in \Delta\right\}=\emptyset$ and $\mathcal{G}$ be the family of all finite subsets of $\Delta$. Suppose $\{\hat{\gamma}$ $\left.\operatorname{pint}\left(V_{\lambda}\right): \lambda \in \delta\right\} \neq \emptyset$ for each $\delta \in \mathcal{G}$. Then $\mathcal{F}=\left\{\cap\left\{\hat{\gamma}-\operatorname{pint}\left(V_{\lambda}\right): \lambda \in \delta\right\}: \delta \in \mathcal{G}\right\}$ becomes a filter base on $X$. Therefore $\mathcal{F}, \hat{\gamma}$-pre- $\theta$-accumulates at some point $x$ of $X$. Again since $\left\{X-V_{\alpha}: \alpha \in \Delta\right\}$ is a $\hat{\gamma}$-preopen cover of $X, x \in X-V_{\alpha_{0}}$ for some $\alpha_{0} \in \Delta$. Let $W=X-V_{\alpha_{0}}$, then we get $W \in \hat{\gamma}-P O(X, x)$ and $\hat{\gamma}-\operatorname{pint}\left(V_{\alpha_{0}}\right) \in \mathcal{F}$ such that $\hat{\gamma}-p c l(W) \cap \hat{\gamma}-\operatorname{pint}\left(V_{\alpha_{0}}\right)=\emptyset$ - a contradiction.

$(\mathrm{d}) \Rightarrow(\mathrm{a})$ : Suppose the family $\left\{V_{\alpha}: \alpha \in \Delta\right\}$ is a $\hat{\gamma}$-preopen cover of $X$. 
Then $\left\{X-V_{\alpha}: \alpha \in \Delta\right\}$ is a family of $\hat{\gamma}$-preclosed subsets of $X$ having empty intersection. So there exists $\alpha_{1}, \alpha_{2}, \ldots, \alpha_{n} \in \Delta$ such that $\cap_{i=1}^{n} \hat{\gamma}-\operatorname{pint}\left(X-V_{\alpha_{\mathrm{i}}}\right)=$ $\emptyset$ i.e. $\cup_{i=1}^{n} \hat{\gamma}-p c l\left(V_{\alpha_{\mathrm{i}}}\right)=X$. Hence $X$ is $\hat{\gamma}$-p-closed.

Theorem 4.6. Let $X$ be a topological space and $S \subset X$. Then the following conditions are equivalent:

(a) $S$ is $\hat{\gamma}$-p-closed relative to $X$;

(b) every ultrafilter base on $X$ which meets $S, \hat{\gamma}$-pre- $\theta$-converges to some point of $S$;

(c) every filter base on $X$ which meets $S, \hat{\gamma}$-pre- $\theta$-accumulates at some point of $X$;

(d) for every family $\left\{V_{\alpha}: \alpha \in \Lambda\right\}$ of $\hat{\gamma}$-preclosed subsets of $X$ such that $\left[\left\{V_{\alpha}\right.\right.$ : $\alpha \in \Lambda\}] \cap S=\emptyset$, there exists finite number of indices $\alpha_{1}, \alpha_{2}, \ldots, \alpha_{n} \in \Lambda$ such that $\left[\cap_{i=1}^{n} \hat{\gamma}-\operatorname{pint}\left(V_{\alpha_{\mathrm{i}}}\right)\right] \cap S=\emptyset$

Proof. The proof is similar to proof of Theorem 4.5 and thus omitted.

Theorem 4.7. Let $X$ be a topological space and $S \subset X$. Then the following conditions are equivalent:

(a) $S$ is $\hat{\gamma}$-strongly compact relative to $X$;

(b) every ultrafilter base on $X$ which meets $S, \hat{\gamma}$-pre-converges to some point of $S$;

(c) every filter base on $X$ which meets $S, \hat{\gamma}$-pre-accumulates at some point of $X$;

(d) for every family $\left\{V_{\alpha}: \alpha \in \Lambda\right\}$ of $\hat{\gamma}$-preclosed subsets of $X$ such that $\left[\left\{V_{\alpha}\right.\right.$ : $\alpha \in \Lambda\}] \cap S=\emptyset$, there exists finite number of indices $\alpha_{1}, \alpha_{2}, \ldots, \alpha_{n} \in \Lambda$ such that $\left[\cap_{i=1}^{n} V_{\alpha_{\mathrm{i}}}\right] \cap S=\emptyset$

Proof. The proof being similar to proof of Theorem 4.5 omitted.

Theorem 4.8. A $\hat{\gamma}$-strongly pre-regular $\hat{\gamma}$-p-closed topological space is $\hat{\gamma}$-strongly compact.

Proof. Let $X$ be a $\hat{\gamma}$-pre-regular $\hat{\gamma}$-p-closed topological space. Suppose $\left\{U_{\alpha}\right.$ : $\alpha \in \Delta\}$ be a $\hat{\gamma}$-preopen cover of $X$. So, for each $x \in X$, there exists $\alpha(x) \in \Delta$ such that $U_{\alpha(x)} \in \hat{\gamma}-P O(X, x)$ and $V_{x} \in \hat{\gamma}-P O(X, x)$ such that $x \in V_{x} \subset \hat{\gamma}$ $\operatorname{pcl}\left(V_{x}\right) \subset U_{\alpha(x)}$. Then $\left\{V_{x}: x \in X\right\}$ is a $\hat{\gamma}$-preopen covering of $X$ and since $X \hat{\gamma}$-p-closed, there exists finite number of points $x_{1}, x_{2}, \ldots, x_{n} \in X$ such that $X=\cup_{i=1}^{n} \hat{\gamma}-p c l\left(V_{x_{\mathrm{i}}}\right) \subset \cup_{i=1}^{n} U_{\alpha\left(x_{\mathrm{i}}\right)}$. Therefore $X$ is $\hat{\gamma}$-strongly compact.

Theorem 4.9. In a $\hat{\gamma}$-p-closed topological space $X$, every $\hat{\gamma}$-pre- $\theta$-closed subset is $\hat{\gamma}$-p-closed relative to $X$. 
Proof. Let $S$ be a $\hat{\gamma}$-pre- $\theta$-closed subset of $X$ and $\left\{U_{\alpha}: \alpha \in \Delta\right\}$ be a cover of $S$ by $\hat{\gamma}$-preopen subsets of $X$. Now, since $S$ is $\hat{\gamma}$-pre- $\theta$-closed, for each $x \in(X-S)$ there exists a $V_{x} \in \hat{\gamma}-P O(X, x)$ such that $\hat{\gamma}-p c l\left(V_{x}\right) \cap S=\emptyset$. Then the family $\left\{U_{\alpha}: \alpha \in \Delta\right\} \cup\left\{V_{x}: x \in X-S\right\}$ is $\hat{\gamma}$-preopen cover of $X$. Again, since $X$ is $\hat{\gamma}$-p-closed, then there exists finite number of indices $\alpha_{1}, \alpha_{2}, \ldots, \alpha_{m} \in \Delta$ and finite number of points $x_{1}, x_{2}, \ldots, x_{n} \in(X-S)$ such that $X=\left[\cup_{i=1}^{m} \hat{\gamma}-p c l\left(U_{\alpha_{\mathrm{i}}}\right)\right] \cup\left[\cup_{j=1}^{n} \hat{\gamma}-\operatorname{pcl}\left(V_{x_{\mathrm{j}}}\right)\right]$ and so $S \subset \cup_{i=1}^{m} \hat{\gamma}-p \operatorname{cl}\left(U_{\alpha_{\mathrm{i}}}\right)$. Hence $S$ is $\hat{\gamma}$-p-closed relative to $X$.

Remark 4.10. In a $\hat{\gamma}$-strongly-compact topological space $X$, every $\hat{\gamma}$ preclosed subset is $\hat{\gamma}$-strongly compact relative to $X$.

Theorem 4.11. Let $f: X \rightarrow Y$ be a quasi $\left(\hat{\gamma}, \hat{\gamma}^{\prime}\right)$-pre-irresolute function and $S$ is $\hat{\gamma}$-strongly compact relative to $X$. Then $f(S)$ is $\hat{\gamma}^{\prime}-p$-closed relative to $Y$.

Proof. Let $\Omega$ by any filter base on $Y$ meeting $f(S)$. Then $f^{-1}(\Omega)=\{A \subset$ $X: f(A) \in \Omega\}$ is a filter base on $X$ meeting $S$. Now since $S$ is $\hat{\gamma}$-strongly compact relative to $X$, by Theorem $4.7, f^{-1}(\Omega) \hat{\gamma}$-pre- $\theta$-accumulates at some point $x \in S$. If possible suppose that $f(S)$ is not $\hat{\gamma}$-p-closed relative to $Y$. Then $\Omega$ does not $\hat{\gamma}$-pre- $\theta$-accumulates at $f(x)$ and so there is a $V \in \hat{\gamma}-P O(Y, f(x))$ and a $F \in \Omega$ such that $\hat{\gamma}-p c l(V) \cap F=\emptyset$. Again since $f$ is quasi $\left(\hat{\gamma}, \hat{\gamma}^{\prime}\right)$-pre-irresolute function, there exist an $U \in \hat{\gamma}-P O(X, x)$ such that $f(U) \subset \hat{\gamma}-p c l(V)$. Therefore $f(U) \cap F \subset \hat{\gamma}-p c l(V) \cap F=\emptyset$ implies $U \cap f^{-1}(F)=\emptyset$ - a contradiction.

Remark 4.12. The image of an $\hat{\gamma}$-strongly compact set relative to $X$ under a $\left(\hat{\gamma}, \hat{\gamma}^{\prime}\right)$-pre-irresolute function $f: X \rightarrow Y$ is $\hat{\gamma}^{\prime}$-strongly compact set relative to $Y$.

Definition 4.13. A function $f: X \rightarrow Y$ is said to be almost $\left(\hat{\gamma}, \hat{\gamma}^{\prime}\right)$-pre$\theta$-closed, if image of each $\hat{\gamma}$-preclosed set is $\hat{\gamma}^{\prime}$-pre- $\theta$-closed.

Theorem 4.14. Let $f: X \rightarrow Y$ be a quasi $\left(\hat{\gamma}, \hat{\gamma}^{\prime}\right)$-pre-irresolute function, where $X$ is $\hat{\gamma}$-strongly compact and $Y$ is Urysohn. Then $f$ is almost $\hat{\gamma}$-pre- $\theta$ closed.

Proof. Let $S$ be a $\hat{\gamma}$-preclosed set in $X$, then by Remark 4.10, $S$ is $\hat{\gamma}$-strongly compact relative to $X$. Therefore, by Theorem 4.11, $f(S)$ is $\hat{\gamma}^{\prime}$-p-closed relative to $Y$. Let $y \in Y-f(S)$, then for each $z \in f(S)$, there exist open sets $U_{z}$ and $V_{z}$ in $Y$ containing $y$ and $z$ respectively such that $\operatorname{cl}\left(U_{z}\right) \cap \operatorname{cl}\left(V_{z}\right)=\emptyset$ i.e. $\hat{\gamma}^{\prime}$ $\operatorname{pcl}\left(U_{z}\right) \cap \hat{\gamma}^{\prime}-\operatorname{pcl}\left(V_{z}\right)=\emptyset$. Then $\left\{V_{z}: z \in f(S)\right\}$ is a cover of $f(S)$ by $\hat{\gamma}^{\prime}$-preopen sets of $Y$. Since $f(S)$ is $\hat{\gamma}^{\prime}$-p-closed relative to $Y$, there exist finite number of 
points $z_{1}, z_{2}, \ldots, z_{n} \in f(S)$ such that $f(S) \subset \cup_{i=1}^{n} \hat{\gamma}^{\prime}-p c l\left(V_{z_{\mathrm{i}}}\right) \subset \hat{\gamma}^{\prime}-p c l\left(\cup_{i=1}^{n} V_{z_{\mathrm{i}}}\right)$. Let $U=\cap_{i=1}^{n} U_{z_{\mathrm{i}}}$, then $U$ is an open and hence $\hat{\gamma}^{\prime}$-preopen set of $Y$ containing $y$. Therefore $\hat{\gamma}^{\prime}-p c l(U) \cap f(S)=\emptyset$ and consequently $y \notin \hat{\gamma}^{\prime}-p c l_{\theta}(f(S))$. Hence $f(S)$ is $\hat{\gamma}^{\prime}$-pre- $\theta$-closed i.e. $f$ is almost $\left(\hat{\gamma}, \hat{\gamma}^{\prime}\right)$-pre- $\theta$-closed.

\section{References}

[1] Abd El-Aziz Ahmed Abo-Khadra, On Generalized Forms of Compactness, Master's Thesis, Faculty of Science, Tanta University, Egypt, 1989.

[2] A. V. Arhangelski and P. J. Collins, On submaximal spaces, Topology Appl., 64 (3) (1995), 219-241.

[3] R. H. Atia, S. N. El-Deeb, and I. A. Hasanein, A note on strong compactness and Sclosedness, Matematicki Vesnik, 6 No. 1 (1982), 2328.

[4] B. M. Uzzal Afsan and C. K. Basu, Some new class of spaces and associated spaces, Jour. of Adv. Research in Pure Math. 4 No. 2 (2012), 59-75.

[5] C. K. Basu, B. M. Uzzal Afsan and M. K. Ghosh, A class of functions and separation axioms with respect to an operation, Hacet. J. Math. Stat., 38, No. 2 (2009), 103-118.

[6] S. N. El-Deeb, I. A. Hasanein, A. S. Mashhoura and T. Noiri, On p-regular spaces, Bull. Math. de la Soc. Sc. Math. de la R.S. de Roumanie Tome., 27, No. 75 (1983), 311-315.

[7] M. E. Abd El-Monsef and A. M. Kozae, Some generalized forms of compactness and closedness, Delta J. Sci., 9 (1985), 257-269.

[8] J. Dontchev, M. Ganster and T. Noiri, On p-closed spaces, Internat. J. Math. Math. Sci., 24 (2000), 203-2012.

[9] J. Dugundji, Topology, Allyn and Bacon, Boston, Mass (1966).

[10] D. S. Janković, On functions with $\alpha$-closed graphs, Glas. Math., Ser III, 18 (1983), 141-148.

[11] A. Kar and P. Bhattacharyya, Some weak separation axioms, Bull. Cal. Math. Soc., 82 (1990), 415-422.

[12] S. Kasahara, Operation compact spaces, Math. Japonica, 24 No. 1 (1979), 97-105.

[13] G. S. S. Krishnan and K. Balachandran, On $\gamma$-semiopen sets in topological spaces, Bull. Cal. Math. Soc., 98 No. 6 (2006), 517-530.

[14] S. N. Maheshwari and S. S. Thakur, $\alpha$-compact spaces, Bull. Inst. Math. Acad. Sinica., 13 No. 4 (1985), 341-347.

[15] S. R. Malghan, and G. B. Navalagi, Almost p-regular, p-completely regular and almost pcompletely regular spaces, Bull. Math. de la Soc. Sci. (R.S.R.) Tome, 34, No. 82 (1990), 317-326.

[16] A. S. Mashhour, M. E. Abd El-Monsef and S. N. El-Deeb, On precontinuous and weak precontinuous mappings, Proc. Math. Phys. Soc. Egypt, 53 (1982), 47-53.

[17] A. S. Mashhour, M. E. Abd El-Monsef and I. A. Hasanein, On pretopological spaces, Bull. Mathe. de la Soc. Math. de la R. S. de Roumanie, Tome, 28 No. 76 (1984). 
[18] A. S. Mashhour, M. E. Abd El-Monsef, I. A. Hasainen and T. Noiri, Strongly compact spaces, Delta J. Sci., 8 (1984), 30-46.

[19] H. Ogata, Operations on topological spaces and associated topology, Math. Japonica, 36 No. 14 (1991), 175-184.

[20] I. L. Reilly and M. K. Vamanamurty, On $\alpha$-continuity in topological spaces, Acta Math. Hungar., 45, No 1-2 (1985), 27-32.

[21] M. K. Singal and A. Mathur, On nearly-compact spaces, Boll. Un. Mat. Ital., 4 No. 2(1969), 702-710.

[22] S. Willard, General Topology, Addition-Wesley (1970). 